\title{
El perfil del emprendedor y los estudios relacionados a los emprendedores Iberoamericanos
}

\author{
The profile of the entrepreneur and studies related to the Latin American \\ Entrepreneurs
}

\section{Sebastián Il Sung $\operatorname{Park}^{1}$ y Sergio Duarte Masi²}

Resumen: La falta de trabajo y la pobreza son problemas comunes en Iberoamérica. Siendo las pequeñas empresas las de mayor fuente creadora de trabajo en la región, surge la importancia de fomentar la labor del emprendedor para atacar la pobreza. La presente revisión bibliográfica explora las teorias relacionadas al perfil del emprendedor y las investigaciones hechas sobre el mismo en Iberoamérica. Del análisis de estos estudios, se destacan algunas caracteristicas que refieren a las principales cualidades del emprendedor, como ser: personas innovadoras, creativas, que ven las oportunidades donde los demás solo perciben la rutina, tolerancia al fracaso, perseverancia, necesidad de logro, motivación, optimismo, trabajo arduo, concentración y disfrutar siendo emprendedor. Se observa también la importancia de las investigaciones sobre el emprendedor de cada pais, debido a las diferentes situaciones que se encuentran en el entorno externo y la cultura de cada uno de ellos.

Palabras clave: Emprendedor, perfil del emprendedor, Iberoamérica.

Abstract: Joblessness and poverty are common problems in Latin America. Being the small enterprises the most creative source of labor in the region, the importance of encouraging entrepreneurs to attack poverty arises. This literature review explores the theories related to the profile of the entrepreneur and the research done on it in Latin America. The analysis of the studies on the entrepreneur, highlights some features that refer to their qualities, such as: innovative, creative people who can see opportunities where others perceive only routine, failure tolerance, perseverance, need for achievement, motivation, optimism, hard work, concentration and enjoy being an entrepreneur. The importance of the study of entrepreneurship in each country is also observed due to the different situations found in the external environment and culture of each one.

Keywords: Entrepreneur, the entrepreneurial profile, Iberoamerican.

\footnotetext{
1 Doctor en Administración de Empresas y Máster en Gestión de Empresas, Docente universitario de la Universidad Autónoma de Asunción. Paraguay. (fotoartpy@hotmail.com)

2 Doctor en Ciencias de la Educación y Ciencias Empresariales y Máster en Ciencias de la Ingeniería. Docente universitario de la Universidad Nacional del Este. Paraguay. (sedumapy@gmail.com)

Recibido: 30/12/2014; Aceptado: 19/01/2015. http://dx.doi.org/10.18004/riics.2015.diciembre.291-314
} 


\section{INTRODUCCIÓN}

Para una mejor comprensión, nos remitiremos al concepto del emprendedor definido por distintos autores. Con respecto al origen de la palabra "emprendedor" encontramos tres versiones. La primera de ellas corresponde a lo señalado por Poncio (2010):

... el término emprendedor proviene de los vocablos latinos in, en, y préndere, cuyo significado es "acometer o llevar a cabo". Por extensión y probablemente por influencia del francés y del italiano, lenguas en las que el empresario se dice entrepreneur e imprenditore respectivamente, el término también se utiliza para señalar a quién inicia una empresa. En consecuencia, puede afirmarse que emprendedor es quien aborda la aventura de un negocio, lo organiza, busca capital para financiarlo y asume toda o la mayor acción de riesgo (p. 21).

La segunda versión se remonta al siglo XVI, cuando "se comenzó a llamar emprendedor a toda persona que, como Cristóbal Colón, se arrojaba a la aventura de viajar al nuevo mundo, sin ninguna certeza de lo que su viaje le deparaba, e incluso sin saber si lograrian volver" (Giurfa, 2012, p. 7).

La tercera de estas versiones indica que su origen data del siglo XVIII, cuando Cantillon utilizó la palabra francés entrepreneur (traducción al español: pionero) en el año 1755 en el texto "Essai Sur la Nature du Commerce en Général", para referirse a aquellos "...quienes eran capaces de asumir el riesgo y la responsabilidad de poner en marcha y llevar a conclusión un proyecto" (Toledo Nickels, 2001, p. 213). Cantillon definió a dicho "pionero" como "un agente económico que compra medios de producción a determinado precio, a fin de combinarlos y crear un nuevo producto" (Giurfa, 2012, pp. 7-8).

Más tarde, otros economistas como Say (1852), Knight (1921), Baumol (1968), Braudel (1985), Casson (1985) continuaron dándole forma al concepto de emprendedor distinguiéndolo del inversionista, ya que las expectativas con respecto de las acciones de cada uno, difieren. El inversionista busca hacer rendir su capital. En cambio, el emprendedor pretende quitar de las utilidades que su enprendimiento genera, los costos generados por el uso del capital del inversionista. Esta discrepancia se enfatizó con el aporte de Schumpeter (1961) quien identificó al emprendedor como el "destructor creativo" que rompe los ciclos del mercado. 
De las diversas definiciones de "emprendedor", podemos extraer algunas características comunes que se distinguen del resto por su gran poder de precisión conceptual. Entre estos rasgos fuertes que precisan el significado de "emprendedor", destacamos las siguientes: personas innovadoras, creativas, que ven las oportunidades donde los demás solo perciben la rutina, el riesgo excesivo y hasta el fracaso y que pueden tomar sus actividades empresarias casi como un juego de azar, en donde el que más apuesta, aún a conciencia de poder perderlo todo, es el que más gana.

Podemos encontrar en la actualidad, numerosos ejemplos de emprendedores que alcanzaron no solo el éxito económico momentáneo sino también aquellos cuyo trabajo ha trascendido de la mera novedad de corto alcance a la innovación que perdura en el tiempo, por su capacidad de renovar su contexto tecnológico y económico social. En esta oportunidad recordaremos, solamente a modo de casos destacados, a los creadores de los software operativos, los programas de navegación por Internet y las redes sociales que han revolucionado en estas últimas dos décadas el ámbito informático.

La presente revisión bibliográfica pretende explorar sobre las teorias relacionadas al perfil del emprendedor y las investigaciones hechas sobre el emprendedor en Iberoamérica, considerando que el espíritu del emprendedurismo es la clave para crear fuente del trabajo sostenible.

\section{Teorias relacionadas al emprendedor}

Existen diversos enfoques con respecto a las distintas teorias relacionadas al tema de los emprendimientos. Para el presente estudio, analizaremos la clasificación hecha por Veciana (1999) quien considera cuatro enfoques teóricos: 1) económico, 2) psicológico, 3) socio-cultural o del entorno y 4) gerencial. Sobre esta base, podemos decir que cada enfoque enfatiza distintas perspectivas del tema incluyendo teorias que los representan. A continuación, explicaremos brevemente cada una de ellas:

1. Enfoque económico: Explica el fenómeno de la creación de empresas debido a la racionalidad económica a nivel de empresario, empresa o sistema económico. Algunas de sus teorias son:

Teoria del cuarto factor de producción: Los autores Say (1840), Mill (1848) y Marshall (1890) conceden mucha relevancia al empresario como "el cuarto factor 
de producción" distinto del terrateniente, del trabajador y del capitalista. De acuerdo a esta teoría, el empresario tomará en arriendo la tierra del terrateniente, las capacidades del trabajador y los bienes de capital del capitalista, para combinarlos en la agricultura, la industria y el comercio.

Teoria del beneficio del empresario: Cantillon (1755), Knight (1921) y Weber (1921) buscan justificar la función y el "beneficio del empresario" a partir de la incertidumbre y el riesgo. El empresario asume el riesgo de adquirir medios de producción y organizar la actividad económica esperando obtener un beneficio al finalizar el proceso productivo. La decisión de convertirse en empresario se basaba únicamente en la propensión o la aversión al riesgo.

Teoría del desarrollo económico: Schumpeter (1934), entiende a los emprendedores como personas que promueven nuevas combinaciones o innovaciones y los ve como los principales promotores del desarrollo económico y los avances sociales. A este proceso lo clasifica en tres partes: invención, innovación e imitación. Sitúa al empresario como elemento dinámico, activo, innovador y quebrantador del equilibrio en el sistema de mercado, al organizar de diferente manera la producción. La esencia del "desarrollo económico" está en la función innovadora $\mathrm{y}$ en las combinaciones productivas que efectúan los empresarios creando nuevas empresas rompiendo de ese modo la rutina de la vida económica.

Teoría de los costes de transacción: Intenta explicar la creación de empresas en base a sus costes. Los costes de transacción vendrian a ser los costos asociados a la utilización y el cálculo del mecanismo de precios de mercado o los costes en los que las empresas incurren cuando, en vez de usar sus propios recursos internos, salen al mercado para encontrar esos productos y servicios. Sus principales representantes fueron Coase (1937) y Williamson (1971).

2. Enfoque psicológico: Analiza al empresario como persona, su perfil y las condiciones de los empresarios exitosos, centrándose en sus características personales. Algunas de las teorías más representativas, son:

Teoría de los rasgos de personalidad: Autores como Lévesque et al. (2002), Djankov et al. (2006) y otros han estudiado los rasgos de personalidad característicos de los emprendedores como factores imprescindibles para poder crear una empresa. Christersen (1994) selecciona los principales atributos de los emprendedores realizando un recorrido histórico. Cada uno de estos autores 
recopilados señala la característica que consideran fundamental y que define a un emprendedor, como podemos apreciar en la tabla 1.

Tabla 1. Principales atributos del emprendedor.

\begin{tabular}{ll}
\hline Autor & Atributo \\
\hline Stuart Mill (1848) & Tolerancia al riesgo \\
Weber (1917) & Fuente de autoridad formal \\
Schumpeter (1934) & Innovación e iniciativa \\
Sutton (1954) & Responsabilidad \\
Hartman (1959) & Autoridad formal \\
McClelland (1961) & Tolerancia al riesgo y necesidad de logro \\
Davids (1963) & Ambición, independencia. Responsabilidad y autocon \\
& fianza. \\
Palmer (1971) & Asunción y control del riesgo \\
Winter (1973) & Afán de poder \\
Borland (1974) & Control percibido interno \\
Liles (1974) & Necesidad de logro \\
Gasse (1977) & Orientado por valores personales \\
Timmons (1978) & Autoconfianza, orientado a la innovación \\
Sexton (1980) & Energla, capacidad de enfrentarse a los problemas \\
Welsh y White (1981) & Necesidad de logro, asunción de riesgos, responsabili \\
& dad y compromiso \\
Dunkelgerg y Cooper (1982) & Independencia, orientado al crecimiento \\
Fernal y Solomon (1986) & Orientado por los valores personales \\
Winslow y Solomon (1987) & Ligeramente sociopático \\
\hline
\end{tabular}

Fuente: Christersen (1994).

Teoría del empresario de Kirzner: La teoría del empresario de Kirzner (1979) define el espíritu emprendedor como el estado de alerta a las oportunidades, basado en las diferencias de las percepciones individuales. Explica el autor que el valor del emprendedor está en el aprovechamiento de las oportunidades de beneficio sin explotar, procedentes de una mala asignación de recursos donde se generan situaciones inciertas, de desequilibrio.

3. Enfoque socio-cultural y del entorno: Examina los factores sociales, políticos, familiares y la influencia del apoyo institucional en la decisión del emprendedor al crear su propia empresa. Algunas de sus teorias, son:

Teoría de la marginación: Esta teoría tiene en cuenta los factores externos, especialmente los negativos. Considera que una empresa se inicia con un suceso crítico, generalmente negativo, que la precipita, también denominado deterioro del rol (Collins et al., 1964) o suceso disparador (Shapero, 1982). Individuos marginados que pueden formar parte de grupos minoritarios étnicos, religiosos, inmigrantes o desempleados, son los más propensos a crear su propia empresa a partir de esos factores negativos y críticos. Según esta teoría para convertirse en empresario son necesarias dos condiciones (Brunet y Alarcón, 2004): un periodo de 
incubación, de maduración de la idea desde hace algún tiempo; y un suceso disparador que provoque el comienzo de la creación de la empresa, sin responder a la intención de obtener un beneficio sino más bien a un factor crítico considerado negativo.

Teoría del rol: La teoría del rol explica porqué en ciertas zonas geográficas se encuentra más cantidad de empresas que en otras. Esto se produce debido a que el entorno en el que predominan modelos o actividades empresariales estimula el surgimiento de más empresarios (Nueno, 1996). Al advertir que individuos en circunstancias similares crearon sus propias empresas, el nuevo empresario se siente confiado en hacer lo mismo (Veciana, 1996). Aunque las caracteristicas del emprendedor son vitales, los factores externos como la presencia de empresarios experimentados en la zona y de modelos de roles empresariales de éxito en la comunidad, tienen un efecto igualmente notorio en la decisión de crear una empresa (Begley y Boyd, 1987). Los valores de la sociedad y las experiencias empresariales en el pasado, con sus éxitos y sus fracasos, legitiman la actividad en el presente, en cuanto que actúa como incentivo en el proceso de creación de empresas. Un aumento de legitimación social implica cambios en las preferencias individuales.

Teoría de redes: Esta teoría tomó importancia en la década de los ochenta, a partir del impacto de la red (network) en la creación de empresas. Diversas investigaciones como las de Birley (1985) y Aldrich et al. (1986) explotaron este fenómeno. La teoría de redes plantea que el conjunto de relaciones específicas entre varios grupos o actores proporciona múltiples interconexiones y reacciones en cadena, cuyo resultado es hacer circular la información y las ideas, y facilitar al emprendedor la creación de la empresa. Para que surjan empresarios dentro del entorno de una red, es indispensable concebir una estructura de organización apropiada, en la cual se especifique el medio más favorable para que ocurran diferentes tipos de interacción (Baba e Imai, 1993). La interacción entre las empresas genera nueva información económicamente valiosa dando lugar a lo que se conoce como aprendizaje mediante la interacción (Ollé y Planellas, 2003).

Teoría de la incubadora: La teoría de la incubadora expresa que la circunstancia que influye primordialmente en la decisión de un individuo que pone en marcha un negocio, proviene de la organización de la que formó parte como trabajador o de la institución en la estuvo siendo formado para tal fin. La base de la teoria de la incubadora se encuentra en la adquisición de las habilidades o destrezas necesarias por parte del futuro empresario en situaciones previas, que le 
sirvan para crear posteriormente su empresa (Bull y Willar, 1993). Diversas investigaciones que analizan el fenómeno de las incubadoras de empresas suelen establecer una conexión entre las organizaciones empresariales, en las que ha estado trabajando el emprendedor con anterioridad, y la nueva empresa, explicando que aquéllas sirven como incubadoras del nuevo proyecto empresarial, dado que es donde el emprendedor descubre la oportunidad de negocio y madura su idea hasta materializarla (Aldrich y Wiedenmayer, 1993).

Teoría de la ecología de la población: Esta teoría planteada por autores como Hannan y Freeman (1977) y Baumol (1993), busca explicar los factores que afectan el ritmo al que nacen las nuevas organizaciones dentro de una población de organizaciones existentes, las cuales compiten por el mismo grupo de recursos del ambiente. Considera que el éxito en la creación de empresas está determinado por el entorno más que por la propia habilidad del empresario. Las empresas que se adapten al entorno sobrevivirán, y las que no lo hagan, morirán. Con respecto a esta teoría, Veciana (1988) indica que las formas de organización existentes en un momento determinado son incapaces de adaptarse a los cambios del entorno; que los cambios del entorno generan nuevas empresas; y que los cambios en las poblaciones de empresas obedecen a procesos demográficos de creación y disolución de éstas.

Teoría institucional: Esta teoría explica cuáles son los aspectos sociales e institucionales que llevan a emprender. Mientras las instituciones proveen el entorno para favorecer el crecimiento económico, el emprendedor será el mecanismo que haga que este crecimiento ocurra. Esta teoría parte de la base de que las instituciones constituyen las reglas de juego en la sociedad, como fuerzas restrictivas impuestas por el hombre que configuran la interacción humana (North, 1990).

4. Enfoque gerencial: Observa el desarrollo en el interior de la empresa una vez establecida focalizándose en las características de la misma y de su organización. Estas teorías no buscan explicar el proceso de creación de empresas sino el papel que desempeña el empresario en él.

Teoría de la eficiencia-X de Leibenstein: La teoría de la eficiencia-X de Leibenstein (1966) analiza el rol del emprendedor. Con el término "eficiencia-X" se refiere al grado de ineficiencia en el uso de recursos dentro de la empresa, midiendo los fracasos de la misma respecto a sus posibilidades productivas. La eficiencia-X surge debido a que los recursos de la empresa son utilizados de una 
forma errónea, porque se consumen de manera equivocada o directamente no se consumen. La falta de esfuerzos de otras personas y la consecuente ineficacia de las organizaciones que los emplean, crean oportunidades para los emprendedores. Los emprendedores eficientes son una amenaza para las organizaciones ineficientes, convirtiéndose en una presión externa que funciona como factor motivador para que estas empresas busquen maximizar sus recursos (Casson, 1991).

Intrapreneurship o Corporate Entrepreneurship: Esta teoria analiza la iniciativa emprendedora que desarrolla dentro de la empresa. Un intraemprendedor es un individuo que ejerce como emprendedor pero dentro de una organización. De acuerdo a Sharma y Chrisman (1999), el Intrapreneurship o Corporate Entrepreneurship es un proceso de renovación empresarial donde se pueden crear nuevos negocios innovando en productos, procesos y tecnologías, dando un nuevo significado al proceso de negocios.

\section{Perfil del emprendedor en Iberoamérica}

Kantis (2004) realizó una reflexión sobre el origen sociocultural y género del emprendedor de América Latina, expresando que en la gran mayoría de los estados actuales la primera matriz de la que nacen los emprendedores es la de la clase media (más del 80\%). Es por esto que las sociedades con una gran porción de sectores medios tienen a su disposición una base potencialmente más amplia para la creación de emprendedores que aquellas más polarizadas. Esto señala una discrepancia entre América Latina y los restantes países del mundo desarrollado, caracterizados -más allá de sus diferencias- por una estratificación social más moderada y por un mayor predominio de la clase media en la población. Por lo tanto, las bases sociales de las cuales suelen surgir los emprendedores son más angostas en América Latina. Adicionalmente, de acuerdo a las investigaciones del autor, en la mayoría de los estados latinoamericanos la presencia relativa de emprendedores dinámicos nacidos de familias de sectores más bajos es más circunscrita (inferior a 25\%).

Otro problema detectado por Kantis (2004) acerca del emprendedor de América Latina, es que las mujeres no participan mayormente en la creación de empresas dinámicas, representando solo uno de cada diez emprendedores. Respecto a la motivación y la dimensión sociocultural para el emprendedor en América Latina, generalmente un familiar constituye la fuente inspiración fundamental. 
Por su parte, Toledo Nickels (2001) se enfoca en la destreza del emprendedor, puesto que la misma se sostiene en la práctica anticipatoria del futuro para crear un plan vital que se hará realidad. La imaginación, la perspectiva de diseñar algo en rigor no existente, es parte significativa de la capacidad del emprendedor. Puede con su pensamiento figurarse el suceso final como ya consumado y a continuación elabora mentalmente la sucesión secuencial de los hechos para lograr su consecución. Este proceso, de acuerdo al autor, no es del todo consciente y probablemente en su principio haya motivaciones emocionales y hasta viscerales.

También Alcaraz Rodríguez (2011) describe las particularidades de los emprendedores y algunas caracterizaciones. En primer lugar, dice que para ser creativo es forzoso contar con una sensibilidad específica para los inconvenientes (propios y ajenos). Asimismo, hay que poseer fluidez de pensamiento o ideas fértiles, así como la capacidad para completarlas y emplearlas en las circunstancias o el problema estudiado. Esta elasticidad puede ser espontánea, cuando es una habilidad para plantear una gran diversidad de ideas, o adaptativa cuando la orientamos en la solución de una temática determinada.

Además de las características ya aludidas, el autor considera que los individuos con pensamiento creativo poseen las siguientes características:

"Fluidez de ideas y capacidad para jugar con ellas. Flexibilidad de pensamiento. Capacidad de juicio y de adaptación ante cualquier situación. Originalidad e imaginación. Sensibilidad hacia los problemas. Espíritu de investigación. Uso adecuado y provechoso de los errores. Disposición para tomar riesgos. Altas autoestima y autosuficiencia. Alto grado de curiosidad. Su conocimiento es base de ideas nuevas. Disposición para el uso de metáforas y analogías en la solución de problemas. Se rigen por criterios estrictos y exigentes. Procuran lograr un buen equilibrio entre subjetividad y objetividad. Aceptan con facilidad la confusión y la incertidumbre. Pueden dejar a un lado los compromisos emocionales. Son capaces de considerar diferentes puntos de vista sobre un mismo tema. No se apresuran para conducir una obra determinada. Están orientados a la autocrítica y aceptan con facilidad las observaciones de los demás" (Alcaraz Rodríguez, 2011, p. 8).

Por su parte, Puchol (2012), efectuó el estudio del emprendedor señalando sus cualidades, las cuales serían: “Adaptabilidad, Asertividad, Asunción de riesgos, 
Autonomia, Creatividad, Comunicación, Concentración, Cooperación, Determinación, Flexibilidad, Independencia de juicio, Intuición, Motivación de logro, Objetividad, Orientación al equipo, Perseverancia, Planificación, Presencia física, Realismo, Responsabilidad, Técnica, Tenacidad y Visión” (Puchol, 2012, p. 26).

En cuanto a los rasgos psicológicos, Alonso Nuez (2011), destaca lo siguiente: "Necesidad de logro o de autorrealización, Control interno y autoconfianza, Espíritu de riesgo o propensión a asumir riesgos, Necesidad de poder e independencia, Compromiso, Tolerancia a la ambigüedad" (Alonso Nuez, 2011, p. 15).

También Poncio (2010) cita las condiciones para ser emprendedor de la siguiente manera: Perseverancia, Independencia, Visión optimista, Pasión, Necesidad de realización y reconocimiento, Vocación innovadora, Autoestima, saber rodearse de buena gente, Autonomía, Iniciativa, Voluntad para aprender.

Basado en las publicaciones estudiadas sobre el perfil del emprendedor podemos resumir de ellas las cualidades esenciales que debe tener dicho empresario, como ser: Asunción de riesgos, Autonomía, Autoestima, Autoconfianza, Creatividad, Enérgico, Flexibilidad, Independencia de juicio, Iniciativa, Motivación/necesidad de logro, Perseverancia, Responsabilidad, Tolerancia a la ambigüedad y Visión optimista.

\section{Estudios relacionados a los emprendedores iberoamericanos}

Para efectuar la revisión de los estudios relacionados al fenómeno de los emprendedores, hemos recurrido a fuentes secundarias como libros, revistas científicas, artículos académicos y otros.

En las mismas, hemos encontrado numerosas investigaciones sobre los emprendedores iberoamericanos $y$ hemos realizado una selección que consideramos acorde a la temática de nuestro trabajo. Los trabajos analizados proceden de España (9), Argentina (5), Brasil (2), Costa Rica (2), México (1), Colombia (1) y Venezuela (1) (Tabla 2). 
Tabla 2. Estudios sobre emprendedores iberoamericanos.

\begin{tabular}{|c|c|c|c|}
\hline Tema & Pais & Autor & Titulo de la investigación \\
\hline \multirow{10}{*}{ 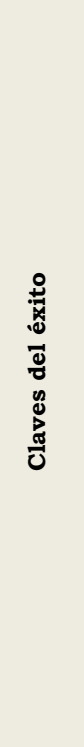 } & \multirow{6}{*}{ España } & $\begin{array}{l}\text { Manzano García y Ayala Calvo } \\
(2007)\end{array}$ & $\begin{array}{l}\text { ¿Puede la psicología positiva ayudar a comprender el } \\
\text { comportamiento del emprendedor? }\end{array}$ \\
\hline & & $\begin{array}{l}\text { Martin Cruz, Barahona y } \\
\text { Rodriguez E.. (2005) }\end{array}$ & $\begin{array}{l}\text { Análisis de la formación y la experiencia laboral como } \\
\text { determinantes del espiritu emprendedor de los } \\
\text { estudiantes universitarios. }\end{array}$ \\
\hline & & $\begin{array}{l}\text { Ruiz Arroyo, Bojica Bojica, } \\
\text { Albacete Sáez y Fuentes Fuentes } \\
\text { (2009) }\end{array}$ & $\begin{array}{l}\text { ¿Cómo identifican las oportunidades de negocio los } \\
\text { emprendedores?: Una aproximación descriptiva al caso } \\
\text { Andaluz. }\end{array}$ \\
\hline & & $\begin{array}{l}\text { Ruiz Navarro, Camelo Ordáz y } \\
\text { Codura Martinez (2012) }\end{array}$ & $\begin{array}{l}\text { Mujer y desafio emprendedor en España. Características } \\
\text { y determinantes. }\end{array}$ \\
\hline & & $\begin{array}{l}\text { García Ramos, Martínez, } \\
\text { Campillo y Fernández Gago } \\
(2010)\end{array}$ & $\begin{array}{l}\text { Caracteristicas del emprendedor influyentes en el } \\
\text { proceso de creación empresarial y en el éxito esperado. }\end{array}$ \\
\hline & & Ripollés y Blesa (2006) & $\begin{array}{l}\text { Redes personales del empresario y orientación } \\
\text { emprendedora en las nuevas empresas. }\end{array}$ \\
\hline & $\begin{array}{l}\text { Venezu } \\
\text { ela }\end{array}$ & Bilbao y Pachano (2002) & Rasgos y actitudes de los emprendedores. \\
\hline & Brasil & Saboia Leitão y Cruz (2006) & $\begin{array}{l}\text { Los rasgos psicológicos del emprendedor y la } \\
\text { continuidad del proyecto empresarial: Un estudio } \\
\text { empirico de emprendedores brasileños. }\end{array}$ \\
\hline & Mexico & $\begin{array}{l}\text { Vargas Hernández y Campos } \\
\text { Alvarez (2011) }\end{array}$ & $\begin{array}{l}\text { Habilidades emprendedoras en los jóvenes } \\
\text { universitarios zacatecanos. }\end{array}$ \\
\hline & Colombia & Fracica Naranjo (2010) & Gerencia Emprendedora para el siglo XXI. \\
\hline \multirow{6}{*}{ 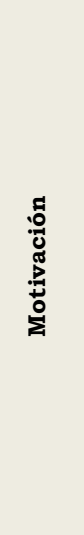 } & \multirow{3}{*}{ España } & $\begin{array}{l}\text { Sánchez, Lanero y Yurrebaso } \\
\text { (2005) }\end{array}$ & $\begin{array}{l}\text { Variables determinantes de la intención emprendedora } \\
\text { en el contexto universitario. }\end{array}$ \\
\hline & & $\begin{array}{l}\text { Coque Martínez, Díaz Bretones y } \\
\text { López Mielgo (2013) }\end{array}$ & $\begin{array}{l}\text { Factores para la puesta en marcha y el éxito de } \\
\text { microempresas asociativas creadas por jóvenes } \\
\text { egresados universitarios. }\end{array}$ \\
\hline & & Justo (2008) & $\begin{array}{l}\text { La influencia del género y entorno familiar en el éxito y } \\
\text { fracaso de las iniciativas emprendedoras. }\end{array}$ \\
\hline & \multirow[t]{2}{*}{$\begin{array}{l}\text { Costa } \\
\text { Rica }\end{array}$} & Leiva Bonilla (2008) & $\begin{array}{l}\text { ¿Nacen empresas de las actividades de fomento al } \\
\text { espiritu emprendedor?: Un vistazo a empresas surgidas } \\
\text { del Concurso Nacional de Emprendedores y el Programa } \\
\text { de Formación en Espiritu Emprendedor del Instituto } \\
\text { Tecnológico de Costa Rica. }\end{array}$ \\
\hline & & Brenes Leiva (2007) & $\begin{array}{l}\text { Emprendedores costarricenses revelando algunos de sus } \\
\text { secretos. }\end{array}$ \\
\hline & Brasil & De Castro e Costa Rizzato (2012) & $\begin{array}{l}\text { Dimensiones de personalidad, motivación de logro y } \\
\text { expectativas de control en jóvenes emprendedores } \\
\text { brasileños. }\end{array}$ \\
\hline \multirow{5}{*}{ 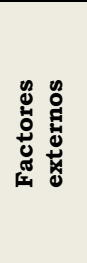 } & \multirow{5}{*}{$\begin{array}{l}\text { Argenti } \\
\text { na }\end{array}$} & Poncio (2010) & Animarse a emprender \\
\hline & & Kantis, (2004) & $\begin{array}{l}\text { Desarrollo emprendedor: América latina y la experiencia } \\
\text { internacional. }\end{array}$ \\
\hline & & Graña (2002) & $\begin{array}{l}\text { Creación de empresas: Factores asociados al éxito } \\
\text { emprendedor en cinco ciudades argentinas. }\end{array}$ \\
\hline & & $\begin{array}{l}\text { Entrialgo, Fernández y Vázquez } \\
(2010)\end{array}$ & $\begin{array}{l}\text { El comportamiento emprendedor y el éxito de la PYME: } \\
\text { Modelos de contingencia y configuracionales. }\end{array}$ \\
\hline & & Quintana García (2001) & Dimensiones del éxito de las empresas emprendedoras, \\
\hline
\end{tabular}


Estas investigaciones se concentran principalmente en el perfil de los emprendedores pero también se focalizan en otros aspectos. Con respecto a las claves del éxito, encontramos estudios de España, Venezuela, Brasil, México y Colombia:

En España:

- Manzano García y Ayala Calvo (2007) en ¿Puede la psicología positiva ayudar a comprender el comportamiento del emprendedor?, estudiaron el comportamiento del emprendedor desde la perspectiva de la Psicología Positiva dando explicación a las causas que llevan al éxito o al fracaso. Buscando la explicación desde una perspectiva multilateral, en la que tenga cabida tanto las características del emprendedor como las peculiaridades del contexto en el que se desenvuelve, la investigación se concentra en la mente positiva de los emprendedores, que lleva a la felicidad y buena vida, destacando la característica del emprendedor; que es, no tener miedo al fracaso.

- Martín Cruz, Barahona y Rodríguez Escudero (2005) en Análisis de la formación y la experiencia laboral como determinantes del espiritu emprendedor de los estudiantes universitarios, buscaron la relación entre el espíritu emprendedor con la formación y la experiencia laboral. Basado en una población de 2012 de estudiantes universitarios, quienes se encontraban en los últimos años de sus estudios. Encontraron que las mujeres muestran menor propensión a crear empresas que los hombres; la edad no presentó un efecto sobre la actitud emprendedora; es importante el conocimiento de informática y la formación en otro tipo de materias; la experiencia laboral previa explica de forma importante la propensión a crear empresas, aunque es diferente para cada sexo.

- Ruiz Arroyo, Bojica Bojica, Albacete Sáez y Fuentes Fuentes (2009) en ¿Cómo identifican las oportunidades de negocio los emprendedores?: Una aproximación descriptiva al caso andaluz, estudiaron los factores y características que influyen en el proceso emprendedor, concretamente en la fase de identificación de oportunidades emprendedoras, basándose en una muestra de 241 empresarios andaluces. En este estudio la mitad de los encuestados expresó que la principal motivación para la creación del negocio fue el autoempleo o la necesidad económica, mientras que el resto afirmó que el factor determinante fue el reconocimiento de una oportunidad. En el resultado ante la cuestión de si son capaces de reconocer oportunidades en sectores donde no poseen experiencia previa casi un $45 \%$ de los encuestados estuvieron de acuerdo, en mayor o menor 
grado. Por último, se consultó acerca de la necesidad de poseer experiencia o conocimientos previos. Casi un $80 \%$ de los encuestados estuvieron de acuerdo.

- Ruiz Navarro, Camelo Ordáz y Codura Martinez (2012) en Mujer y desafio emprendedor en España. Características y determinantes, estudiaron a las características y determinantes del emprendimiento femenino en España y arribaron a las siguientes conclusiones: el conocimiento de otros emprendedores facilita el proceso emprendedor y la red de contactos en tamaño y diversidad; es más intenso en el colectivo masculino; presenta una menor tendencia al endeudamiento que en los hombres; tiene menor presencia en actividades intensivas en capital; y una menor propensión a asumir riesgos.

- García Ramos, Martínez, Campillo y Fernández Gago (2010) en Características del emprendedor influyentes en el proceso de creación empresarial y en el éxito esperado, estudiaron a los factores individuales del emprendedor que pueden condicionar su iniciativa empresarial y el éxito esperado. El estudio se realizó con una muestra de 19.384 individuos con residencia en España y llegó a la conclusión de que las características más relevantes del emprendedor influyente son: no tener miedo al fracaso; poder identificar buenas oportunidades de negocio en el entorno; poseer los conocimientos y habilidades necesarios para crear y dirigir una nueva empresa y; una edad próxima a los 40 años.

- Ripollés y Blesa (2006) en Redes personales del empresario y orientación emprendedora en las nuevas empresas, realizaron la revisión de las características de las redes personales (tamaño, densidad, composición, frecuencia de contacto y diversidad) que inciden positivamente sobre el crecimiento de las ventas. La muestra estaba formada por 500 nuevas empresas pertenecientes a sectores diversos ubicadas en la comunidad valenciana. Este estudio se concentró en la influencia de las redes personales en las nuevas empresas al analizar su papel como proveedoras de recursos intangibles esenciales, valiosos y críticos capaces de proporcionar a las nuevas empresas ventajas competitivas sostenibles. Las conclusiones a las que se arribó se relacionan con la importancia de concientización de las redes personales, que constituye una fuente de recursos muy valiosos para el desarrollo de nuevas oportunidades de negocio.

En Venezuela:

- Bilbao y Pachano (2002) en Rasgos y actitudes de los emprendedores, estudiaron a las cualidades de emprendedores en Venezuela, con el fin de apoyar a 
la eficacia del Proyecto Andino de Competitividad, desarrollado por la Corporación Andina de Fomento. La muestra estaba formada por 53 empresas de la base de datos de la institución Venezuela Competitiva y los resultados obtenidos concluyeron que muchas de las competencias encontradas en los estudios internacionales coinciden con las encontradas en los emprendedores venezolanos. Asimismo, se observaron tres importantes estrategias que aplican las empresas con buen desempeño: todas ellas consideran de gran importancia la innovación y la aplican constantemente en los productos y procesos; todas ellas dedican parte de sus ganancias para invertirla en mejoras internas; y ninguna descuida la capacitación y el adiestramiento, aún en momentos de crisis.

En Brasil:

- Saboia Leitão y Martín Cruz (2006) en Los rasgos psicológicos del emprendedor y la continuidad del proyecto empresarial: Un estudio empirico de emprendedores brasileños, buscaron comprender a los emprendedores brasileños mediante el enfoque psicológico. Utilizando una muestra formada por 1.448 individuos que realizaron el programa EMPRETEC (Brasil), llegaron a las siguientes conclusiones: los rasgos psicológicos inciden en la supervivencia de un proyecto empresarial; los emprendedores con más creatividad, capacidad de innovación, liderazgo y respaldo por parte de familiares, amigos e instituciones son aquellos cuyos proyectos tienen más posibilidades de sobrevivir; es de suma importancia la formación y experiencia; y cuanto antes ha empezado el emprendedor a tener experiencias profesionales, mayor es la posibilidad de que su proyecto sobreviva.

En México:

- Vargas Hernández y Campos Alvarez (2011) en Habilidades emprendedoras en los jóvenes universitarios zacatecanos, estudiaron a la efectividad en el desarrollo de las habilidades emprendedoras de los jóvenes en base a los programas de fomento emprendedor de las universidades públicas en Zacatecas, Fresnillo y Guadalupe. Se utilizó el muestreo aleatorio simple, que se aplicó en los alumnos que cursaran los Programas de Desarrollo de Emprendedores (PDE) en determinadas universidades mexicanas. Este estudio señala como características del emprendedor las siguientes: compromiso, determinación y perseverancia; capacidad para alcanzar metas; orientación a las metas y oportunidades; iniciativa y responsabilidad; persistencia en la solución de problemas; realismo; con auto confianza; altos niveles de energía; buscador de retroalimentación; con un control 
interno alto; tomador de riesgos calculados; baja necesidad de estatus y poder; integro y confiable; tolerante al cambio.

En Colombia:

- Fracica Naranjo (2010) en Gerencia Emprendedora para el siglo XXI, realizó la caracterización de la gerencia emprendedora y establecimiento de los requisitos para su implementación en las organizaciones. El estudio caracteriza al intraempresario desde el punto de vista de la personalidad, de las relaciones y de trabajo. Llega a la conclusión de que: a) Desde el punto de vista de la personalidad es proactivo y construye su desarrollo integral; se gerencia a sí mismo; b) Desde el punto de vista de las relaciones, ve en los problemas oportunidades y desarrolla un sentido de pertenencia con la organización; genera confianza, relaciones de mutuo apoyo e interdependencia; c) Desde el punto de vista de trabajo, muestra una actitud favorable hacia el cambio, está dispuesto a realizar su trabajo de manera diferente, no tiene temor de ensayar nuevas alternativas, de equivocarse y de aprender de los errores; hacen su trabajo con pasión.

Otros trabajos, procedentes de España, Costa Rica y Brasil, buscan específicamente las cualidades del emprendedor relacionado con la motivación del mismo. Estos son:

En España:

- Sánchez, Lanero y Yurrebaso (2005) en Variables determinantes de la intención emprendedora en el contexto universitario, desarrollaron un modelo explicativo de la intención emprendedora considerando variables de tipo personal. Los datos se recogieron de una muestra de 320 varones y 574 mujeres constituida por estudiantes universitarios, por considerarse ésta una población de potenciales emprendedores. Los resultados obtenidos confirman la existencia de una relación positiva directa entre la personalidad proactiva y las intenciones emprendedoras.

- Coque Martínez, Díaz Bretones López Mielgo (2013) en Factores para la puesta en marcha y el éxito de microempresas asociativas creadas por jóvenes egresados universitarios, analizan los motivos y otros factores que facilitan la puesta en marcha y el éxito de empresas colectivas creadas por universitarios teniendo en cuenta el papel desempeñado por las universidades. El análisis empírico consistió en el estudio de 13 empresas creadas por egresados de tres universidades españolas. Los resultados muestran que el desarrollo profesional y valores 
personales como iniciativa, independencia, capacidad de adaptación y constancia son las motivaciones más señaladas por los universitarios para crear empresas con sus compañeros. Asimismo, la universidad aparece como un elemento de apoyo a través de asignaturas que colaboran con la formación de futuros emprendedores.

- Justo (2008) en La influencia del género y entorno familiar en el éxito y fracaso de las iniciativas emprendedoras, estudió a los emprendedores desde la perspectiva de género y entorno familiar. La muestra se conformó con datos obtenidos en el proyecto Global Entrepreneurship Monitor (GEM) España que cuenta con 40 países de todo el mundo. Sus principales hallazgos fueron: el género no influye a la dimensión intrínseca del éxito; existe un efecto directo del género en la percepción del éxito como independencia; existe un efecto directo del género en la decisión voluntaria del cierre empresarial; las mujeres emprendedoras no valoran las metas intrínsecas en mayor medida que los emprendedores; el efecto de género si se activa con la presencia de hijos dependientes. Respecto a la importancia dada por las mujeres emprendedoras a la independencia como meta no-económica, los emprendedores y las emprendedoras con hijos dependientes que dirigen una empresa establecida tenderán a adherirse a los estándares del éxito financiero y de valoración de lo económico.

En Costa Rica:

- Leiva Bonilla (2008) en ¿Nacen empresas de las actividades de fomento al espiritu emprendedor?: Un vistazo a empresas surgidas del Concurso Nacional de Emprendedores y el Programa de Formación en Espiritu Emprendedor del Instituto Tecnológico de Costa Rica, estudió el proceso de creador de empresas entre las personas que participaron en el Concurso Nacional Emprendedor y el Programa de Formación en Espíritu Emprendedor en Costa Rica. La muestra estuvo conformada por 131 personas distribuidas en tres grupos: emprendedores activos (aquellos que ya tienen su empresa funcionando), emprendedores nacientes (quienes están haciendo gestiones pero no han iniciado como empresa formal) y no emprendedores (personas que no tienen su empresa ni planean hacerlo en el corto o mediano plazo). Los datos sobre los emprendedores activos y nacientes muestran que se requiere seguir inculcando desde el sector educativo un cambio de actitud en cuanto al emprendimiento, si se quiere incrementar el número de emprendedores que toman el riesgo de lanzar su empresa. Algunas acciones concretas en este campo es enfocar buena parte de los cursos sobre espíritu emprendedor, que ya existen en las universidades nacionales, hacia el fomento de actitudes o motivaciones y no solamente a la escritura de planes de negocios. 
- Brenes Leiva (2007) en Emprendedores costarricenses revelando algunos de sus secretos, describió la situación de los emprendedores de Costa Rica. La muestra estuvo conformada por cincuenta emprendedores costarricenses a quienes se les consultó sobre las razones que los impulsaron a crear su negocio, los mayores obstáculos que enfrentaron y las satisfacciones que vivieron. Se concluyó que para lograr el éxito en los negocios es necesario mucho esfuerzo, trabajo arduo, dedicación y sentir pasión por lo que se hace. Igualmente, la perseverancia, el compromiso, el no ser conformista, actuar de manera positiva y gozar de apoyos.

En Brasil:

- De Castro e Costa Rizzato (2012) en Dimensiones de personalidad, motivación de logro y expectativas de control en jóvenes emprendedores brasileños investigaron las caracteristicas de la personalidad que configuran el perfil del sujeto emprendedor. La población de la investigación fueron de 475 estudiantes universitarios Manaus, Brasil. En su estudio clasifican al sujeto de investigación en tres categorías: Soñadores, Seguidores y Emprendedores. Las principales conclusiones fueron que el grupo de los Emprendedores poseían el más alto grado en responsabilidad en todas sus facetas; el más bajo en neuroticismo sobre todo en las facetas de depresión, vulnerabilidad y ansiedad social; son más activos y asertivos, más abiertos a nuevas ideas e innovadores; están más motivados por el logro que por los resultados; sus estrategias de afrontamiento elegidas para resolver los problemas con estrés son el afrontamiento activo, la planificación y la reinterpretación positiva; tienen una mayor creencia en que son ellos quienes manejan su vida, percibiéndose como menos indefensos ante la adversidad, más auto eficaces y con más altas expectativas de éxito.

Los estudios encontrados sobre las influencias de los factores externos al emprendedor, se encuentran las siguientes investigaciones:

En Argentina:

- Poncio (2010) en Animarse a emprender, elaboró una guía que los emprendedores desarrollen sus actividades de producción y comercialización, puedan acceder al financiamiento necesario de acuerdo a sus posibilidades económicas, apropiarse de las tecnologías adecuadas, disponer de condiciones de precio justo y consumo responsable, y formalizar su emprendimiento para de esa manera disponer de obra social y seguridad social. 
- Kantis (2004) en Desarrollo emprendedor: América latina y la experiencia internacional, exploró el campo de la creación de empresas y aporta ideas originales y respuestas concretas obtenidas de un estudio basado en la experiencia de 2000 fundadores de empresas jóvenes de países de América Latina, Italia, España y Este del Asia, así como también las principales tendencias internacionales en materia de iniciativas de apoyo a la creación de empresas y estudios de casos de buenas prácticas de desarrollo emprendedor en distintas regiones.

- Graña (2002) en Creación de empresas: Factores asociados al éxito emprendedor en cinco ciudades argentinas, estudió el proceso de implementación de ideas de negocio en cinco ciudades de Argentina y los elementos que diferencian a los emprendimientos que logran transitar exitosamente la etapa de gestación de una nueva empresa. El trabajo de campo fue realizado en cinco localidades de Argentina con personas que estuvieran realizando acciones para iniciar una nueva empresa. El tamaño de la muestra aproximado fue de 2100 hogares en cada ciudad. En cuanto a los aspectos personales de los emprendedores consultados, se destacaron el carácter de propensión al riesgo y la autoconfianza.

- Entrialgo, Fernández y Vázquez (2010) en El comportamiento emprendedor y el éxito de la PYME: Modelos de contingencia y configuracionales, realizaron el análisis de la naturaleza del comportamiento emprendedor y su relación con la estrategia, el entorno industrial y el éxito de la empresa. Basado en el estudio de 233 empresas españolas, el estudio afirma que el emprendedor toma una decisión caracterizado por las dimensiones de innovación, asunción de riesgos y proactividad.

- Quintana García (2001) en Dimensiones del éxito de las empresas emprendedoras, estudió a las dimensiones explicativas del éxito de los proyectos emprendedores de Andalucia, España. La muestra estuvo conformada por un grupo de empresas de rápido crecimiento de la región de Andalucía, España. Este estudio realizó una revisión de la teoría económica, teoría de la organización y dirección estratégica acerca de la trascendencia de la complejidad externa y la influencia organizativa en la supervivencia y éxito empresarial; y elaboró una taxonomía de los factores determinantes del proceso de creación y desarrollo de empresas. El factor considerado más importante es la red de apoyo moral proporcionada por los familiares y amigos. En la dimensión interna, la mayoría de las empresas tenía claramente delimitado desde el inicio de la actividad el nicho del mercado que constituía su objetivo. La financiación es la principal preocupación en la etapa de rápido crecimiento. 
Como puede notarse, los diversos estudios mencionados sobre el emprendedor, refieren principalmente sobre el perfil de los emprendedores, que nos permite comprender las cualidades del mismo desde distintos puntos de vista y diversos paradigmas.

\section{COMENTARIOS}

Hemos comenzado nuestro trabajo indagando acerca de las múltiples teorías que existen actualmente relacionadas a los emprendedores y las circunstancias que los rodean. Fueron analizadas desde cuatro enfoques: económico, psicológico, socio-cultural y del entorno y gerencial, lo que nos permitió visualizar el tema desde distintas perspectivas. Asimismo, hemos explorado en investigaciones sobre el perfil del emprendedor, donde hemos podido extraer algunas características que se destacan al referirse a las cualidades del emprendedor, tales como: personas innovadoras, creativas, que ven las oportunidades donde los demás solo perciben la rutina, tolerancia al fracaso, perseverancia, necesidad de logro, motivación, optimismo, trabajo arduo, concentración y disfrutar siendo emprendedor.

De las investigaciones analizadas en los países de Iberoamérica, hemos podido percatarnos también de las diferencias existentes entre los países de Iberoamérica en su calidad y cantidad de estudio sobre el emprendedor. La cantidad y la calidad de las investigaciones tienen una relación directa con el nivel de desarrollo de cada país. Inclusive la infraestructura para fomentar al emprendimiento tendria la misma relación. De lo expuesto, se puede inferir que la falta de estudio sobre el emprendedor y la falta de infraestructura para el fomento del emprendedor llevan a la falta del trabajo. Y la falta del trabajo induce a la pobreza del país, la cual completando un ciclo vicioso, por ende lleva a la falta de estudio y de infraestructura para el emprendimiento.

Por tanto, destacamos la importancia del estudio sobre emprendedurismo y la necesidad de realizar el estudio particular de cada país, debido a las diferencias existentes en el entorno externo y la cultura de cada país. Sugerimos fomentar el estudio sobre la situación del emprendedor como parte de la lucha contra la pobreza.

\section{REFERENCIAS}

Alcaraz Rodríguez, R. (2011). El emprendedor de éxito. $4^{\text {a }}$ Ed. México: Mc Graw Hill. 
Aldrich, H.; Rosen, B. y Woodward, W. (1986). Social Behaviour and Entrepreneurial Networks, En Ronstadt, H. et. al., Frontiers of Entrepreneurship Research (pp. 239-240). Centre for Entrepreneurial Studies, Babson College.

Aldrich, H.E. y Wiedenmayer, G. (1993). From traits to rates: an ecological perspective on organizational foundings. Greenwich, CT: JAI Press.

Alonso Nuez. M. J. (2011). El emprendedor y la empresa: Una revisión de los determinantes a su constitución, acciones e investigaciones sociales. España: Universidad de Zaragoza.

Baba, Y., e Imai, K. (1993). Una concepción reticular de la innovación y el impulso empresarial: La evolución de los sistemas VCR. Revista Internacional de Ciencias Sociales, 135, 27-37.

Baumol, W. J. (1993). The Entrepreneur an Economic Theory. MIT Press.

Begley, T. y Boyd, D. (1987). Psycological characteristics associated with performance in entrepreneurial firms and small businesses. Journal of Business Venturing, 2, 79- 93.

Bilbao, A. y Pachano, S. (2002). Rasgos y actitudes de los emprendedores: Proyecto andino de competitividad. Caracas, Venezuela: Corporación Andina de Fomento.

Birley, S. (1985). The role of networks in the entrepreneurial process. Journal of Business Venturing, 1, 107-117.

Braudel, F. (1985). Les dynamiques du capitalisme. Paris, Francia: Arthaud.

Brenes Leiva, G. (2007). Emprendedores costarricenses revelando algunos de sus secretos, Tec Empresarial, 1(2), 16-19.

Brunet, I., y Alarcón, A. (2004). Teorías sobre la figura del emprendedor. Papers, 73, 81-103.

Bull, I., y Willard, G. E. (1993). Towards a Theory of Entrepreneurship. Journal of Business Venturing, 8, 183-195.

Cantillon, R. (1755). Ensayo sobre la naturaleza del comercio en general. México: Fondo de Cultura Económica.

Casson, M. (1982). The entrepreneurs: An economic theory. Nueva York: Barnes and Noble.

Christensen, M. A. (1994). The identification of business starters using attitude. Calgary: University of Calgary Press. 
Coase, R. (1937). The nature of the firm. Económica, 4(16).

Collins, O. F., Moore, D.G., y Unwala, D.B. (1964). The organization makers: A behavioural study of independent entrepreneurs. New York: Meredith.

Coque Martínez, J.; Díaz Bretones, F., y López Mielgo, N. (2013). Factores para la puesta en marcha y el éxito de microempresas asociativas creadas por jóvenes egresados universitarios. REVESCO Revista de estudios cooperativos, 81.

Cruz Soto, L. A. (2006). La regularidad del comportamiento administrativo a partir del concepto de dominación de Max Weber. México DF, Mexíco: Red Contaduría y Administración.

De Castro e Costa Rizzato, S. (2012). Dimensiones de personalidad, motivación de logro y expectativas de control en jóvenes emprendedores brasileños (Tesis doctoral). Universidad de León, León, España

Djankov, S.; Qian, y Roland, G., y Zhuravskaya, E. (2006). Who are China's entrepreneurs?, American Economic Revie, 96(2), 348-352.

Entrialgo M., Fernández, E., y Vázquez C. J. (2010). El comportamiento emprendedor y el éxito de la PYME: Modelos de contingencia y configuracionales. Dirección y Organización, Revista de Ingeniería de Organización, 25.

Fracica Naranjo, G. (2010). Gerencia emprendedora para el siglo XXI. Barranquilla, Colombia: Universidad Autónoma del Caribe.

García Ramos, C. G., Martínez C., A., y Fernández Gago, R. (2010). Características del emprendedor influyentes en el proceso de creación empresarial y en el éxito esperado, Revista Europea de Dirección y Economía de la Empresa, 19(2), 42.

Giurfa Johnson, A. F. (2012). Estudio de desarrollo emprendedor de la población joven en la provincia de Tacna, Tacna, Perú: Universidad Privada de Tacna.

Graña, F. M. (2002). Creación de empresas: Factores asociados al éxito emprendedor en cinco ciudades argentinas. Mar del Plata, Argentina: Universidad Nacional de Mar del Plata.

Hannan, M., y Freeman, J. H. (1977). The population ecology of organizations. American Journal of Sociology, 82(5), 86-118.

Justo, R. (2008). La influencia del género y entorno familiar en el éxito y fracaso de las iniciativas emprendedoras. (Tesis doctoral). Universidad Autónoma de Madrid. 
Kantis, H. (2004). Desarrollo emprendedor: América Latina y la experiencia internacional, Washington D.C., Estados Unidos: Banco Interamericano de Desarrollo.

Kirzner, I. M. (1979). Perception, opportunity and profit: Studies in the theory of entrepreneurship. The university of Chicago Press.

Knight, F. H. (1921). Enterprise and profit: En Risk, uncertainly and profit (pp. 264-290). University of Chicago, Chicago.

Leibenstein, H. (1966). Allocative efficiency vs. x-efficiency. American Economic Review, 56, 392-415.

Leiva Bonilla, J. C. (2008). ¿Nacen empresas de las actividades de fomento al espíritu emprendedor?: Un vistazo a empresas surgidas del Concurso Nacional de Emprendedores y el Programa de Formación en Espíritu Emprendedor del Instituto Tecnológico de Costa Rica. Tec Empresarial, 2(1), 16-27.

Lévesque, M., Shepherd, D. A., y Douglas, E. J. (2002). Employment or self-employment: A dynamic utility maximiz- ing model. Journal of Business Venturing, 17, 189-210.

Manzano García, G., y Ayala Calvo, J. C. (2007). ¿Puede la psicología positiva ayudar a comprender el comportamiento del emprendedor. Logroño, España: Universidad Rioja.

Marshall, A. (1890). Principios de Economía. España: Editorial Sintesis S.A.

Martín Cruz, N., Barahona, J. H., y Rodríguez Escudero, A. I. (2005). Análisis de la formación y la experiencia laboral como determinantes del espiritu emprendedor de los estudiantes universitarios. Valladolid, España: Universidad de Valladolid.

Mill, J. S. (1848). Principles of political economy. London, England: Longmans, Green and Co.

North, D. (1990). Institutions, institutional change and economic performance. Cambridge, UK: Cambridge University Press.

Nueno, P. (1996). Evolución de los conceptos de management. Management Review, 1,73-83).

Ollé, M., y Planellas, M. (2003). El proceso de creación de una empresa: Motivaciones, etapas, recursos y redes en Creación de Empresa: Los mejores textos. Ed. Ariel Empresa.

Poncio, D. (2010). Animarse a emprender. Villa María, Argentina: Eduvim Editorial Universitaria. 
Puchol, L. (2012). El libro del emprendedor: Cómo crear tu empresa y convertirte en tu propio jefe. Madrid, España: Ediciones Díaz de Santos.

Quintana García, C. (2001). Dimensiones del éxito de las empresas emprendedoras, Investigaciones Europeas de Dirección y Economia de la Empresa, 7(2), 139-154.

Ripollés M. M., y Blesa P. A. (2006). Redes personales del empresario y orientación emprendedora en las nuevas empresas. Cuadernos de Economía y Dirección de la Empresa, 26, 73-93.

Ruiz Arroyo, M., Bojica Bojica, A. M., Albacete Sáez, C. A., y Fuentes Fuentes, M. (2009). ¿Cómo identifican las oportunidades de negocio los emprendedores?: Una aproximación descriptiva al caso andaluz. Granada, España: Universidad de Granada.

Ruiz Navarro, J., Camelo Ordáz, M., y Codura Martínez, A. (2012). Mujer y desafío emprendedor en España: Características y determinantes. Cádiz: Economía Industrial.

Saboia Leitao, F. A., y Cruz, N. (2006). Los rasgos psicológicos del emprendedor y la continuidad del proyecto empresarial: Un estudio empírico de emprendedores brasileños. León, España: Universidad de León.

Sánchez J. C., Lanero, A., y Yurrebaso, A. (2005). Variables determinantes de la intención emprendedora en el contexto universitario. Revista de Psicología Social Aplicada, 15(1), 37-60.

Say, J. (1852). Première partie. En Traité d'économie politique. Paris, Francia Guillaumin et Cie.

Schumpeter, J. (1961). Entrepreneurial profit: The theory of economic development. Harvard University Press, 4,128-156.

Shapero, A. (1982). Social dimensions of entrepreneurship. En C. Kent et al. (eds.), The encyclopedia of entrepreneurship. Englewood Cliffs, NJ: Prentice Hall.

Sharma, P., y Chrisman, J. J. (1999). Toward a reconciliation of the definitional issues in the field of corporate entrepreneurship. Entrepreneurship Theory and Practice, 23(3), 11-27.

Toledo Nickels, U. (2001). Ejercicio de construcción de un ideal tipo de la vida social: El caso del emprendedor. Red Cinta de Moebio, 12, 209-226.

Vargas Hernández, J. G., y Campos Alvarez, R. E. (2011). Habilidades emprendedoras en los jóvenes universitarios zacatecanos. Ecorfan Journal, 2(5), 70-90. 
Veciana, J. M. (1988). Empresario y proceso de creación de empresas. Revista Económica de Cataluña, 8, 2-34.

Veciana, J. M. (1999). La creación de empresas: Un enfoque empresarial. Colección Estudios Económicos, 33.

Weber, M. (1999). Economía y sociedad: esbozo de la sociología comprensiva. Tr. José Medina Echavarría et al., 2da. Edición en español. México DF, México: Fondo de Cultura Económica.

Williamson, O. E. (1971). The vertical integration of production: market failure considerations. American Economic Review, 61(2), 112-123. 